University of Wollongong

Research Online

Faculty of Social Sciences - Papers (Archive) Faculty of Arts, Social Sciences \& Humanities

2013

Patterns of food safety knowledge among Australians: a latent class approach

Anthony Worsley

Deakin University, tworsley@uow.edu.au

Wei Wang

Deakin University, wwang@swin.edu.au

Stephanie Byrne

Deakin University

Heather Yeatman

University of Wollongong, hyeatman@uow.edu.au

Follow this and additional works at: https://ro.uow.edu.au/sspapers

Part of the Education Commons, and the Social and Behavioral Sciences Commons

Research Online is the open access institutional repository for the University of Wollongong. For further information contact the UOW Library: research-pubs@uow.edu.au 


\title{
Patterns of food safety knowledge among Australians: a latent class approach
}

\begin{abstract}
This study aimed to examine food safety knowledge and its associations among Australians. An Internetbased nationwide survey of 2,022 consumers was conducted in 2011. Quota sampling was used to ensure that the age, gender, educational background, and state of residence of the respondents were representative of the Australian population. A list of 10 food safety knowledge items was administered along with questions about the respondents' food attitudes, demographics, school education, and diet practices. Overall, the results showed that safety knowledge was relatively poor. Latent class analysis identified two groups of respondents with different levels of food safety knowledge. Poor knowledge was negatively associated with age, the female gender, university education, experience of home economics or health education at school, the use of salt reduction diets, and general interest in food issues. The study's limitations and implications are discussed.
\end{abstract}

\section{Keywords}

approach, class, patterns, australians, food, latent, safety, knowledge, among

Disciplines

Education | Social and Behavioral Sciences

\section{Publication Details}

Worsley, A., Wang, W. C., Byrne, S. \& Yeatman, H. (2013). Patterns of food safety knowledge among Australians: a latent class approach. Journal of Food Protection, 76 (4), 646-652. 
Patterns of food safety knowledge among Australians - a latent class approach

Anthony Worsley ${ }^{1}$, Wei. C. Wang ${ }^{1}$, Stephanie Byrne ${ }^{1}$, and Heather Yeatman ${ }^{2}$

${ }^{1}$ School of Exercise and Nutrition Sciences, Deakin University, Australia

${ }^{2}$ School of Health Sciences, University of Wollongong, Australia

*Corresponding author.

Email address: tonyw@deakin.edu.au 


\begin{abstract}
The study aimed to examine food safety knowledge and its associations among Australians. An internet-based nationwide survey of 2022 consumers was conducted in 2011. Quota sampling was used to ensure that the ages, gender, educational background and state of residence were representative of the Australian population. A list of ten food safety knowledge items was administered along with questions about the respondents’ food attitudes, demographics, school education and dieting practices. Overall, the results showed that safety knowledge was relatively poor. Latent class analysis identified two groups of respondents with different levels of food safety knowledge. Poor knowledge was negatively associated with age, female gender, university education, experience of home economics or health education at school, the use of salt reduction diets and general interest in food issues. The study’s limitations and implications are discussed.
\end{abstract}

Key words: Food, Safety, Knowledge, Survey, Australia 
Food borne diseases are a widespread public health issue that can cause serious illness and death. According to the World Health Organisation (WHO), an estimated 2.2 million people die each year from food and water borne diarrhoeal disease worldwide, 1.9 million of whom are children (30). In industrialised nations, food borne diseases affect up to $30 \%$ of the population annually (29). In Australia, there are an estimated 5.4 million cases of food borne illness each year which result in around 1.2 million doctor's visits, 15,000 hospital admissions and 120 deaths and cost the country $\$ 1.25$ billion annually $(4,11,13)$. Recent data suggests that the incidence of foodborne illness worldwide and in Australia is increasing (9).

Food borne illnesses are largely preventable with improved food safety practices. Poor food handling practices in the home cause a significant number of food borne illnesses, however, few studies have investigated the knowledge and food safety practices of consumers in Australia (5, 7, 9, 12, 13, 19, 28). A number of studies in the United Kingdom, the United States, Europe and New Zealand, have demonstrated that many consumers have inadequate food safety knowledge. This includes knowledge of appropriate cooling and storage of cooked foods; safe preparation of meat and vegetables, adequate hand washing; correct refrigerator temperature; cleansing of food preparation surfaces; and awareness of the pathogens which can cause food poisoning $(3,14,23-25)$.

Several studies have found relationships between food safety knowledge and gender, age, education level and completion of a home economics course. A study conducted in Ireland by McCarthy et al. (17) segmented their study sample based on food safety knowledge levels and found that the segment with the lowest knowledge level was more likely to be male, aged 18-24 or 64 and older, have a primary level of education and was less likely to 
have completed a home economics course or read broadsheet newspapers. Research conducted by Langiano et al. (14) in Cassino, Italy, observed that women aged 30-39 were more likely to be more knowledgeable about food safety that men. Sanlier (25) also found a direct relationship between knowledge and age and knowledge and education in a research study conducted in Turkey. No significant relationships were observed, however, between food safety knowledge and gender (25). A U.S. meta-analysis of 20 food safety studies found that men, young adults, those without a high school education and those on high incomes were the least knowledgeable about good hygiene and cross-contamination prevention practices (21).

Australians' levels of food knowledge appear to be similar to those in other populations $(12,13,19,23)$. For example, a study conducted in Melbourne found that $99 \%$ of participants reported at least one poor food safety practice, with the most common being poor cooling and storage of cooked foods and poor surface preparation methods (19). An observational study conducted in 1997 and 1999 found that infrequent and inadequate hand washing and inadequate cleaning of kitchen surfaces were among the most common poor food handling practices performed in Australian home kitchens (12). The study also showed that observed food handling practices were much worse than those reported in a questionnaire prior to the surveillance period (12).

Unfortunately all these studies were conducted more than 10 years ago so there is a need to examine present day Australian consumers’ food knowledge. Further, the characteristics of consumers with low food safety knowledge remain uncertain. This information is critical to allow food safety campaigns to adequately target at risk consumers. 
Therefore, the present study aimed to identify consumers with low food safety knowledge levels who are likely to be at increased risk of food borne illness.

The Food Knowledge Survey conducted at the end of 2011 examined Australian adults' knowledge of a variety of food issues, including food safety. Based on previous research, we hypothesised that age (17), education (25), female gender (14) and completion of home economics education (17) would be positively related to food safety knowledge. We also considered that the presence of children under 5 years would be likely to expose consumers to high food safety awareness, that the use of special diets (e.g. vegetarian, salt reduced and diabetic diets) would indicate greater awareness of food and health issues and so might increase food safety awareness as would interest in food issues generally.

\section{Materials and Methods}

Sampling The Food Knowledge Survey was an internet-based survey conducted nationally during November and December 2011. The survey was conducted by Global Market Insights (GMI), an international market research company. Participants from GMI’s database of registered adults living in Australia were invited by email to participate and provided with a link to the survey. Quota sampling was used to ensure that the ages, gender, educational background and state of residence represented the proportions found in the Australian population. Two thousand and twenty two respondents took part in the survey.

The study was approved by the Deakin University Faculty of Health Human Ethics Committee (HEAG 127-2011). 
Questionnaire The questionnaire was designed to determine Australian adults’ knowledge of a range of issues related to food including the components of a healthy diet, the nutrient content and health consequences of foods, safe food practices, and a variety of environmental and ethical food issues such as animal welfare and climate change. It was a combination of newly created questions and modified questions from earlier studies.

The food safety section of the questionnaire was a selection of modified questions from a validated survey conducted by Byrd-Bredbenner et al. (8) and a questionnaire used by Jay et al. (6). Respondents were questioned about their knowledge of appropriate food handling practices in the areas of ready to eat food preparation, defrosting meat, cleaning dishes and food preparation surfaces, refrigerator temperatures, storing and reheating leftovers, and foods that are not recommended for certain groups in the community (Table 1). For example, Question 1- The best way to prevent food poisoning from fresh fruits and vegetables is to wash them with (Tick one): (a) Regular soap, (b) Hot water, (c) Anti-bacterial soap, (d) An anti-bacterial sponge, and (e) Cool running water. The remaining nine questions used similar response scales to Question 1. If the respondent chose the correct answer, a score of 1 was given and a 0 score represented all the incorrect answers.

Respondents were also asked about their attitudes to a number of food issues including the nutritional properties of foods; cooking and food preparation; food safety; food label reading; the production, processing and distribution of food; food marketing and regulation; food terminology; appropriate serve sizes; the environmental impacts of food production; fair trade; animal welfare; food security and ethical decision-making. The importance of each of the issues was rated using 5 point Likert scales (from Not important (1) to Very important (5)). The responses were summed to form an Attitude score (Cronbach's alpha $=0.95$ ). 
In addition, the survey requested background information about the respondents. This included details of the respondents': age, gender, and education status (high school, technical and trade qualifications, university education); the presence of children under 18 years in their household; whether they were the main food shopper or shared the shopping; whether they had attended school home economics or health courses, or health or food education courses in Years 11 and 12 of secondary school; their consumption of vegetarian, semi vegetarian or vegan diets; and consumption of low salt or diabetes control diets.

Data analysis Latent class analysis (LCA) is similar to factor analysis for continuous variables; it accommodates an analogous approach for measuring categorical latent variables (15). Unlike many multivariate methods, LCA allocates a sample population into mutually exclusive and exhaustive subgroups (10). Like cluster analysis, it attempts to allocate respondents into one or more groups. In the present study, the response patterns of the nine food knowledge items were subjected to LCA to identify the number of classes (or groups) to which the respondents may belong. LCA was carried out with Mplus version 6.1 (20). The maximum likelihood estimation method was used to adjust the standard errors of the present analyses.

The performance of two and three latent class models was assessed. Of these competing latent class models, the selection of the best fitting model was subject to several statistical fit indices as well as theoretical considerations. The Akaike information criterion (AIC, 2) and the Bayesian information criterion (BIC, 26) were used to assess the LCA (15). In addition, the sample size adjusted BIC (aBIC, 27) was used to determine the number of classes from the competing LCA models (31). Several goodness-of-fit measures that incorporate various penalties for model complexity (27) were employed. Smaller values 
indicate better fit. Entropy is a measure of the classification accuracy (22). It can range from 0 to 1 , with higher values indicating better classification. Moreover, higher values of the loglikelihood test statistic suggest better model fit.

The current analysis included the predictors of class membership (15) in which the suitable latent classes were regressed on participants' background characteristics. All of these factors were considered as possible influences of population heterogeneity on the respondents' item responses. The multinomial logistic regression coefficients for each of the classes were then estimated and compared to the reference class via odd ratios.

\section{Results}

The mean age of the participants $(n=2022)$ was $42.6 \pm 14.2$ SD years. Just over half were male (50.4\%), and most participants (59.6\%) were married or living with their partner, and $66.5 \%$ did not have children under 18 years living with them. Just under one third (31.3\%) had a TAFE or trade qualification; and 31.6\% had a university qualification, and 53.8\% had studied home economics and/or health at school. Almost two thirds (61.9\%) were the primary grocery shopper in their household.

Overall the participants’ food safety knowledge was poor. The lowest levels of knowledge related to the areas of freezer temperature, reheating leftovers and washing dishes (29\%, 38\% and 48\% correctly answering these questions, respectively; Table 1). Some areas however, were understood better, for example, the washing of fruits and vegetables, refrigerator temperature, and the defrosting of meat. Generally women performed better than men and most of the safety knowledge was associated with whether the respondents were the 
main shoppers in the households. Respondents' personal background information is presented in Table 2.

Latent class results Table 3 shows the model fit statistics derived from the LCA from the two-class latent class model and the three-class latent class model for men and women when the 10 items of safe and unsafe food practices and covariates were included in the model. Examination of the fit indices and loglikelihood statistics in Table 3 shows that a twoclass solution provided the most parsimonious description for the male and female data. Two latent classes were identified for both men and women. The response probabilities for each of the 10 food safety practices are presented for each of the latent classes in Table 4 . The classification of the two latent classes was based on these response probabilities.

Notably, men and women classified as members of class 1 were more likely to report higher food safety knowledge than their peers in class 2. In other words, class 1 represents those who performed moderately well on the items (moderate knowledge) and class 2 - those who performed poorly (poor knowledge).

The results of the multinomial logistic regression analyses are presented in Table 5. Class 1 (moderate knowledge) is compared with class 2 (poor knowledge) to interpret the effects of the covariates including age, education, main shopper, children's presence at home, studied home economics at school, had food study in year 11 and 12, being on diabetes diet, being on low salt diet, being on vegetarian diet, and attitudes toward food on the latent class membership. The estimated log odds coefficients and the corresponding log odds confidence intervals were then converted into odds ratios and their confidence intervals. 
Table 5 shows the odds ratios and their 95\% intervals. These results suggest that for men as age increases, the odds of being in class 1 (moderate knowledge) versus class 2 (poor knowledge) were over two times higher $(\mathrm{OR}=2.20)$. For men who reported having attended home economics or health classes at school, the odds of being in class 1 (moderate knowledge) versus class 2 (poor knowledge) were nearly two and half times higher than for men who did not attend home economics or health classes at school $(\mathrm{OR}=2.45)$. Men who reported having had undertaken food studies in year 11 and 12, or who were on diabetes diet, or on vegetarian diet were less likely to be in class 1 (moderate knowledge) versus class 2 (poor knowledge). Finally, for men who were on low salt diets, the odds of being in class 1 (moderate knowledge) versus class 2 (poor knowledge) were increased nearly two and half times $(\mathrm{OR}=2.41)$.

Among women, for those who had higher levels of education and more positive attitudes toward food, the odds of being in class 1 (moderate knowledge) versus class 2 (poor knowledge) were one and half times higher than for women who had lower education $(\mathrm{OR}=1.5)$ and negative food attitudes $(\mathrm{OR}=1.48)$. As age increased, the odds of a woman being in class 1 (moderate knowledge) versus class 2 (poor knowledge) were over two times higher $(\mathrm{OR}=2)$. For women who reported having attended home economics or health classes at school, the odds of being in class 1 (moderate knowledge) versus class 2 (poor knowledge) were over three and half times higher than for women who did not attend these classes $(\mathrm{OR}=3.69)$. Moreover, women who reported having undertaken food studies in years 11 and 12, were less likely to be in class 1 (moderate knowledge) versus class 2 (poor knowledge). 


\section{Discussion}

This study showed that respondents' knowledge of the assessed areas of food safety generally was moderate at best, though some areas of food safety were understood better than others. However, the study has also shown that there are two groups of consumers, a substantial minority with poor knowledge and a majority with 'moderate' knowledge of food safety. Furthermore, membership of these two groups was associated with several demographic, educational and behavioral variables.

The poor state of consumers' knowledge, as measured by the items used in this study, is consistent with findings from previous studies in Australia and around the world (12, 19, 21). However, the better levels of knowledge about the washing of fruits and vegetables, defrosting of meat and refrigerator temperatures is important as they suggest that communication activities by government and industry organisations over the past decade may have influenced consumers. However, it is clear that there are low levels of knowledge about some crucial areas such as storing hot meals for several hours, food safety for pregnant women, infants and children, and the reheating of leftovers. The identification of two groups of consumers with contrasting levels of knowledge was made possible through the LCA analysis which is suited to the analysis of categorical data (in this case, true/false items). It provides an elementary segmentation of the population according to food safety knowledge. The multinomial regression results provide some description of the two groups that could be used to tailor communications to them (Table 5).

Some of the characteristics of the group with poor knowledge are familiar to public health workers; they tended to be male, and less educated than those with better knowledge. These men tend to be less motivated to follow prudent health practices and at greater risk of 
health problems than women and higher educated groups (17). The findings, however, go further than identifying the well-known gender/socio economic status divide (16). They show that food safety knowledge is positively related to attitudes to (or interest in) a wide range of food issues, and in varying ways to school educational experience and dietary practices.

This association of safety knowledge with attitudes to food issues is unsurprising since most cognitive behavior models such as the Theory of Planned Behavior (1) posits a strong relationship between attitudes and beliefs. However, the present findings do suggest that food safety knowledge depends on broader interest in food issues; if consumers do not have positive attitudes to food issues then they are less likely to know about food safety. Perhaps communication programs might focus on motivating uninterested consumers to become more interested in food by focusing on issues that motivate them, such as the cost of food or the social acceptability of food.

The age of the respondents was a major predictor of food safety knowledge. The older the respondents were, the better their food safety knowledge. This may be because of the greater experience of people with safety issues as they age and their greater exposure to food safety communications. It might also be due to increasing sensitisation to safety requirements as they age, part of the greater awareness of mortality that is claimed to accompany aging (18). This finding suggests that older people may be more responsive to safety communications and could play roles in the dissemination of food safety information.

The association with exposure to school home economics or health education suggests that school education actually does communicate lasting food safety knowledge. Whilst this finding confirms the that of McCarthy et al. (17), it does require further investigation. The 
possible effects of home economics and health education need to be separated, and more detail of these forms of educational experiences need to be gathered. The negative relationship of secondary school food education in years 11 and 12 is puzzling. It suggests that respondents who had taken these courses were less likely to have moderate food safety knowledge. It may be due to an overemphasis on technological issues in these courses to the detriment of practical safety knowledge. It is important that this finding is confirmed and investigated further.

The observed relationships with dietary practices were largely unexpected. However, together with the food attitudes finding, they show that food safety knowledge is clearly related to dietary practices including diabetes control diets, low salt diets, and vegetarian diets. The relationships of dieting practices with safety knowledge were varied - vegetarian and diabetic diets were negatively related, salt reduction diets were positively linked to better knowledge. Salt reduction may be akin to food safety in that both require attention to technical detail whilst vegetarianism and diabetes diets are major dietary changes that may lead to more concentration on the diets rather than food safety knowledge. The participants who followed a salt reduction diet had significantly more positive attitudes towards food issues than those on normal diets. Therefore, it would be important to identify the motivations of these groups, for example, their interest in prevention of disease or in food preparation more broadly, so that food safety messages could be more carefully targeted. Again, these findings require confirmation in further research.

Implications for food safety communication and policy These findings confirm earlier reports that, with some exceptions, the public's general knowledge about food safety is not high. The main novel findings here are the identification of two knowledge groups and 
the various factors which predict membership of these groups. This suggests that there are at least two target groups of consumers for food safety communications and that these groups have several risk factors which influence their knowledge. Communication programs could be tailored to the needs and interests of these two groups, for example, people on vegetarian and diabetic diets might be explicitly targeted with food safety information. Programs might be designed for younger people as their knowledge is poorer than that of older people, and more emphasis might be given to the teaching of food safety in secondary school health, food and home economics courses.

Consumers’ food preparation behaviors have changed over recent decades. For example, the items related to cleaning of cutting boards that have been adapted from the earlier studies may not be as relevant to current food practices as they once were, for example, today, many people buy chopped or diced raw foods that are ready to cook. Therefore, in future research, we need to examine the safety knowledge that is relevant to current food practices. This implies that we need to monitor food practices regularly.

Limitations and research directions Causal relationships cannot be claimed from the present cross sectional design. Longitudinal monitoring of food safety knowledge is required to establish the causal influences on population food safety knowledge. Such monitoring could be used to evaluate ongoing food safety communication programs. More comprehensive measures of food safety knowledge could be included in future studies. Better assessment of dietary practices and educational experiences is also required. 
Conclusions The public's knowledge of food safety is not high. There are two groups of consumers with low and moderate levels of food safety knowledge. Food safety knowledge is associated with age, genders, school education, food attitudes and dietary practices.

\section{Acknowledgements}

This research was funded by a grant from the Australian Research Council Discovery scheme (DP1094493). The authors express their gratitude to Roxan Toll and Michael Mruczkowski from Global Market Insight, Inc. who administered the survey.

\section{References}

1. Ajzen, I. 1991. The Theory of Planned Behavior. Organizational Behavior and Human Decision Processes. 50:179-211.

2. $\quad$ Akaike, H. 1987. Factor analysis and AIC. Psychometrika. 52:317-332.

3. Altekruse, S. F., D. A. Street, S. B. Fein, and A. S. Levy. 1996. Consumer knowledge of foodborne microbial hazards and food-handling practices. Journal of Food Protection. 59:287-294.

4. Australian Government Department of Health and Ageing. 2005. Foodborne illness in Australia: Annual incidence circa 2000. In Australian Government Department of Health and Ageing, Canberra.

5. Bean, N. H., J. S. Goulding, C. Lao, and F. J. Angulo. 1996. Surveillance for foodborne-disease outbreaks--United States, 1988-1992. MMWR. CDC surveillance summaries : Morbidity and mortality weekly report. CDC surveillance summaries / Centers for Disease Control. 45:1-66.

6. Bravo, A., Y. Cass, and D. Tranter. 2008. Good food in family day care: Improving nutrition and food safety in family day care. Nutrition \& Dietetics. 65:47-55. 
7. Bryan, F. L. 1988. Risks of practices, procedures and processes that lead to outbreaks of foodborne diseases. Journal of Food Protection. 51:663-673.

8. Byrd-Bredbenner, C., V. Wheatley, D. Schaffner, C. Bruhn, L. Blalock, and J. Maurer. 2007. Development and implementation of a food safety knowledge instrument. Journal of Food Science Education. 6:46-55.

9. Food Standards Australia New Zealand. 2012. Incidence of foodborne illness. In Food Standards Australia New Zealand, Caberra.

10. Goodman, L. A. 1974. Exploratory latent structure analysis using both identifiable and unidentifiable models. Biometrika. 61:215-231.

11. Hall, G., M. D. Kirk, N. Becker, J. E. Gregory, L. Unicomb, G. Millard, R. Stafford, K. Lalor, and t. O. W. Group. 2005. Estimating foodborne gastroenteritis, Australia. Emerging Infectious diseases. 11:1257-1264.

12. Jay, L. S., D. Comar, and L. D. Govenlock. 1999. A video study of Australian domestic food-handling practices. Journal of Food Protection. 62:1285-1296.

13. Jay, S. L., D. Comar, and L. D. Govenlock. 1999. A National Australian Food Safety Telephone Survey. Journal of Food Protection. 62:921-928.

14. Langiano, E., M. Ferrara, L. Lanni, V. Viscardi, A. Abbatecola, and E. Vito. 2012. Food safety at home: knowledge and practices of consumers. Journal of Public Health. 20:4757.

15. Lanza, S. T., L. M. Collins, D. R. Lemmon, and J. L. Schafer. 2007. PROC LCA: A SAS procedure for latent class analysis. Structural Equation Modeling. 14:671-694.

16. Marmot, M. 2004. Social determinants of health: A panoramic view. In, International Balzan Foundation, Laudationes, discorsi, saggi. Milan: Libri Scheiwiller. 
17. McCarthy, M., M. Brennan, A. L. Kelly, C. Ritson, M. de Boer, and N. Thompson. 2007. Who is at risk and what do they know? Segmenting a population on their food safety knowledge. Food Quality and Preference. 18:205-217.

18. Meer, R. R., and S. L. Misner. 2000. Food safety knowledge and behavior of expanded food and nutrition education program participants in Arizona. Journal of Food Protection. 63:1725-1731.

19. Mitakakis, T. Z., M. I. Sinclair, C. K. Fairley, P. K. Lightbody, K. Leder, and M. E. Hellard. 2004. Food safety in family homes in Melbourne, Australia. Journal of Food Protection. 67:818-822.

20. Muthén, L. K., and B. O. Muthén. 1998-2012. Mplus user's guide. Muthén \& Muthén. Available as a download at http://www.statmodel.com/ugexcerpts.shtml, Los Angeles, CA. 21. Patil, S. R., S. Cates, and R. Morales. 2005. Consumer food safety knowledge, practices, and demographic differences: Findings from a meta-analysis. Journal of Food Protection. 68:1884-1894.

22. Ramaswamy, V., W. S. Desarbo, D. J. Reibstein, and W. T. Robinson. 1993. An empirical pooling approach for estimating marketing mix elasticities with PIMS data. Marketing Science. 12:103-124.

23. Redmond, E. C., and C. J. Griffith. 2003. Consumer Food Handling in the Home: A Review of Food Safety Studies. Journal of Food Protection. 66:130-161.

24. sanlier, N. 2009. The knowledge and practice of food safety by young and adult consumers. Food Control. 20:538-542.

25. Sanlier, N. 2010. Food safety knowledge and the safe food handling behaviours of female and male consumers. Pakistan Journal of Medical Sciences. 26:653-658.

26. Schwarz, G. 1978. Estimating the dimension of a model. The Annals of Statistics. 6:461-464. 
27. Sclove, S. 1987. Application of model-selection criteria to some problems in multivariate analysis. Psychometrika. 52:333-343.

28. Scott, E. 1996. Foodborne disease and other hygiene issues in the home. Journal of Applied Microbiology. 80:5-9.

29. WHO. 2007. First formal meeting of the Foodborne Disease Burden Epidemiology Reference Group (FERG), 26-28 November 2007. In World Health Organization, Geneva. 30. WHO. 2008. Food safety, zoonoses and food born diseases. In World Health Organization, Geneva.

31. Yang, C. C. 1998. Finite mixture model selection with psychometrics applications. In, vol. PhD. University of Califormia, Los Angeles, CA. 
Table 1

Comparison of men and women's food safety knowledge percentages

\section{Correct (\%)}

Item

Male Female $\chi^{2}(n=2022$, Total

$$
d f=1)
$$

\section{Washing fruits and vegetables}

73.9

81.5

$16.64^{* *}$

77.6

2. Use soapy water and rinse for

45.3

58.8

$8.24 * *$

52.0

cleaning a cutting board

3. Use different cutting board

48.0

39.3

$45.06 * *$

4. Use automatic dishwasher to

40.7

56.1

$32.83 * *$

48.4

washing dishes

5. Refrigerator temperature

65.4

58.6

$9.73^{* *}$

62.0

6. Storing a hot meal for several

55.7

65.2

$18.93^{* *}$

60.4

hours

7. Reheating leftovers

36.8

38.4

.54

37.6

8. Foods safe for pregnant women,

49.6

64.4

$45.45^{* *}$

56.9

infants and children

9. Defrosting meat in fridge

53.7

69.1

$68.87^{* *}$

61.3

10. Defrosting meat in microwave

35.4

25.3

12.59**

30.4 
Table 2

Personal background characteristics across age groups

\begin{tabular}{|c|c|c|c|c|}
\hline \multirow[t]{2}{*}{ Demographics } & & \multirow{2}{*}{$\begin{array}{l}\text { men } \\
(n=1019)\end{array}$} & \multirow{2}{*}{$\begin{array}{l}\text { women } \\
(n=1003)\end{array}$} & \multirow{2}{*}{$\begin{array}{l}\text { Total } \\
(n=2022)\end{array}$} \\
\hline & & & & \\
\hline \multirow[t]{6}{*}{ Age (years) (\%) } & $18-24$ & 12.9 & 14 & 13.4 \\
\hline & $25-34$ & 20.5 & 19.2 & 19.9 \\
\hline & $35-44$ & 23.7 & 19.7 & 21.8 \\
\hline & $45-54$ & 19.7 & 22.1 & 20.9 \\
\hline & $55-64$ & 16.1 & 17.8 & 17 \\
\hline & $65+$ & 7.1 & 7.1 & 7.1 \\
\hline \multirow[t]{7}{*}{ Education (\%) } & Year 11 or & 16.7 & 22.5 & 19.6 \\
\hline & less & & & \\
\hline & Completed & 15.8 & 19.3 & 17.6 \\
\hline & year 12 & & & \\
\hline & TAFE or & 32.4 & 30.1 & 31.3 \\
\hline & trade & & & \\
\hline & University & 35.1 & 28 & 31.6 \\
\hline \multirow[t]{4}{*}{ Main shopper (\%) } & Self & 49.4 & 74.6 & 61.9 \\
\hline & Self and & 33.2 & 18.7 & 26 \\
\hline & partner & & & \\
\hline & Partner & 10.7 & 1.7 & 6.2 \\
\hline Children’s presence (\%) & Yes & 29.9 & 37.2 & 33.5 \\
\hline $\begin{array}{l}\text { Home economics or health } \\
\text { education (\%) }\end{array}$ & Yes & 38.1 & 69.8 & 53.8 \\
\hline Food studies in year 11 \& $12(\%)$ & Yes & 11.9 & 16.8 & 14.3 \\
\hline
\end{tabular}


Food safety knowledge

\begin{tabular}{lllll}
\hline Diabetes control diet (\%) & Yes & 8.4 & 6 & 7.2 \\
\hline Low salt diet (\%) & Yes & 12.2 & 13.7 & 12.9 \\
\hline Vegetarian diet (\%) & Yes & 6.7 & 9.3 & 8 \\
\hline Attitude & & & & \\
\hline
\end{tabular}

Note: $\mathrm{Sd}=$ standard deviation 
Table 3

Criteria to assess model fit for age group specific LCA models with covariates

\begin{tabular}{llllll}
\hline & \multicolumn{2}{l}{ Males } & & & \multicolumn{2}{l}{ Females } \\
\cline { 2 - 3 } \cline { 5 - 6 } Number of classes & 2 class & 3 class & & 2 class & 3 class \\
\cline { 2 - 3 } Loglikelihood & -6027.087 & -5950.683 & & -5196.423 & -5155.893 \\
\# of parameters & 31 & 52 & & 31 & 52 \\
AIC & 12116.173 & 12005.365 & & 10454.847 & 10415.787 \\
BIC & 12268.897 & 12261.547 & & 10607.080 & 10671.146 \\
aBIC & 12170.438 & 12096.390 & & 10508.622 & 10505.990 \\
LMR $p$-value & .0000 & .051 & & .000 & .058 \\
Entropy & .677 & .639 & & .690 & .651 \\
\hline
\end{tabular}

Note: $\mathrm{AIC}=\mathrm{Akaike}$ information criterion, $\mathrm{BIC}=$ Bayesian information criterion, aBIC=sample size adjusted Bayesian information criterion, 
Table 4

Latent class models with covariates across age groups - probability of latent class membership and item response probabilities within each of the four classes

\begin{tabular}{|c|c|c|c|c|}
\hline & \multicolumn{2}{|l|}{ Males } & \multicolumn{2}{|l|}{ Females } \\
\hline & Class1: & Class2: & Class1: & Class2: \\
\hline & high & low & high & low \\
\hline & knowledge & knowledge & knowledge & knowledge \\
\hline Probability of latent class membership & $56.9 \%$ & $43.1 \%$ & $65.4 \%$ & $34.6 \%$ \\
\hline 1. washing fruits and vegetables & 0.90 & 0.52 & 0.87 & 0.72 \\
\hline 2. cutting board - use soapy water and rinse & 0.73 & 0.60 & 0.75 & 0.69 \\
\hline 3. cutting board - use different cutting board & 0.85 & 0.54 & 0.89 & 0.74 \\
\hline 4. wash dishes - use automatic dishwasher & 0.86 & 0.53 & 0.94 & 0.60 \\
\hline 5. refrigerator temperature & 0.75 & 0.52 & 0.62 & 0.53 \\
\hline 6. storing a hot meal for several hours & 0.74 & 0.32 & 0.82 & 0.33 \\
\hline 7. reheating leftovers & 0.47 & 0.24 & 0.50 & 0.16 \\
\hline 8. foods safe for pregnant women, infants and children & 0.63 & 0.33 & 0.77 & 0.41 \\
\hline
\end{tabular}


9. defrost meat-in fridge

10. defrost meat-microwave
0.95

0.86
0.55

0.38
1.00

0.90
0.75

0.40 
Table 5

Estimated odds ratio and 95\% confidence interval between knowledge classes with covariates for gender groups

\begin{tabular}{llll}
\hline & Males & Females \\
\cline { 2 - 3 } Contrast of latent classes & class1 & class1 \\
& vs. & vs. \\
& class2 & class2 \\
\hline 1. age & $2.20(1.80,2.68)^{* *}$ & $2.00(1.67,2.39)^{* *}$ \\
2. education & $1.14(0.94,1.38)$ & $1.50(1.23,1.84)^{* *}$ \\
3. main shopper & $0.99(0.98,1.00)$ & $0.99(0.98,1.00)$ \\
4. children home & $1.03(0.69,1.54)$ & $1.29(0.84,1.97)$ \\
5. home economics at school & $2.45(1.62,3.71)^{* *}$ & $3.69(2.27,5.99)^{* *}$ \\
6. food study in year $11 \& 12$ & $0.44(0.24,0.79)^{* *}$ & $0.52(0.30,0.93)^{*}$ \\
7. diabetes diet & $0.36(0.18,0.70)^{* *}$ & $0.58(0.25,1.35)$ \\
8. low salt diet & $2.41(1.24,4.69)^{*}$ & $1.26(0.65,2.44)$ \\
9. vegetarian diet & $0.40(0.17,0.99)^{*}$ & $0.58(0.29,1.18)$ \\
10. attitude & $1.11(0.88,1.40)$ & $1.48(1.15,1.92)^{* *}$ \\
\hline
\end{tabular}

Note: ${ }^{*} p<.05 ; * * p<.01$ for the multinomial logistic latent class regression weights. 